\title{
DO MODERATE-LUMINOSITY ACTIVE GALACTIC NUCLEI SUPPRESS STAR FORMATION?
}

\author{
Kevin Schawinski $^{1,2}$, Shanil Virani $^{1,2}$, Brooke Simmons $^{1,2}$, C. Megan Urri $^{1,2}$, Ezequiel Treister ${ }^{3,5}$, Sugata Kaviraj $^{4,6}$, \\ AND BRONIKA KUSHKULEY ${ }^{1,2}$ \\ ${ }^{1}$ Department of Physics, Yale University, New Haven, CT 06511, USA; kevin.schawinski@yale.edu \\ ${ }^{2}$ Yale Center for Astronomy and Astrophysics, Yale University, P.O. Box 208121, New Haven, CT 06520, USA \\ ${ }^{3}$ Institute for Astronomy, 2680 Woodlawn Drive, University of Hawaii, Honolulu, HI 96822, USA \\ ${ }^{4}$ Department of Physics, University of Oxford, Oxford OX1 3RH, UK \\ Received 2008 October 16; accepted 2008 December 24; published 2009 January 21
}

\begin{abstract}
The growth of supermassive black holes and their host galaxies are thought to be linked, but the precise nature of this symbiotic relationship is still poorly understood. Both observations and simulations of galaxy formation suggest that the energy input from active galactic nuclei (AGNs), as the central supermassive black hole accretes material and grows, heats the interstellar material and suppresses star formation. In this Letter, we show that most host galaxies of moderate-luminosity supermassive black holes in the local universe have intermediate optical colors that imply the host galaxies are transitioning from star formation to quiescence, the first time this has been shown to be true for all AGNs independent of obscuration. The intermediate colors suggest that star formation in the host galaxies ceased roughly $100 \mathrm{Myr}$ ago. This result indicates that either the AGNs are very long lived, accreting for more than 1 Gyr beyond the end of star formation, or there is a $\sim 100$ Myr time delay between the shutdown of star formation and detectable black hole growth. The first explanation is unlikely given current estimates for AGN lifetimes, so low-luminosity AGNs must shut down star formation before substantial black hole accretion activity is detected. The scarcity of AGN host galaxies in the blue cloud reported here challenges scenarios where significant star formation and black hole growth are coeval. Lastly, these observations also strongly support the "Unified Model" of AGNs as the host galaxy colors are independent of obscuration toward the central engine.

Key words: galaxies: active - galaxies: evolution - galaxies: formation - galaxies: nuclei - X-rays: galaxies
\end{abstract}

\section{INTRODUCTION}

Several works have noted that obscured active galactic nuclei (AGNs) appear to be prevalent in the "green valley" on the color-magnitude diagram (CMD), between actively starforming galaxies in the blue cloud and passively evolving galaxies on the red sequence (Schawinski et al. 2007a; Salim et al. 2007; Nandra et al. 2007; Silverman et al. 2008; Constantin et al. 2008; Bundy et al. 2008; Georgakakis et al. 2008; Schawinski et al. 2009). The presence of AGNs in galaxies with such intermediate optical colors has been interpreted as evidence for the role of AGNs in the suppression of star formation, so that AGN host galaxies are transitioning from the blue cloud to the red sequence. However, the obscuration that affects the central nuclear region might also be reddening the galaxy colors systematically. Therefore, to understand the connection between star formation and black hole growth, we need to study a complete, unbiased sample of AGNs.

In particular, do all AGNs, including both unobscured and highly obscured, Compton-thick AGNs, lie in the green valley, as one might hypothesize from the Unified Model (Urry \& Padovani 1995; Antonucci 1993)? In this Letter, we test the universality of the association of AGNs with "green" optical colors using a complete sample of obscured and unobscured AGN host galaxies at very low redshift and then discuss the implications of the observed optical color distribution for the role of moderate-luminosity AGNs in the suppression of star formation. We include for the first time not just obscured AGNs, but also unobscured and highly obscured sources, and can thus

\footnotetext{
5 Chandra Fellow.

${ }^{6}$ Research Fellow funded by the Royal Commission for the Exhibition of 1851.
}

draw strong conclusions on the host galaxies of AGNs-not just where on the color-magnitude relation they lie, but also where they are absent.

\section{SWIFT BAT SAMPLE SELECTION}

In order to select a complete sample of both unobscured and obscured AGNs, we use hard X-ray selection, which is nearly unbiased with respect to obscuration, yet remains highly efficient, unlike IR selection techniques. The Burst Alert Telescope (BAT) onboard the Swift satellite (Gehrels et al. 2004) observes photons in the $14-195 \mathrm{keV}$ range in which even the most highly obscured AGNs can be detected.

We start with the sample of AGNs found by the Swift BAT in the first nine months of operation across the entire sky (Tueller et al. 2008), then limit ourselves to objects that overlap with the Sloan Digital Sky Survey (SDSS; York et al. 2000; Adelman-McCarthy et al. 2008) and have redshifts of $0.01<z<0.07$, yielding 21 AGNs in the luminosity range of $L_{14-195 \mathrm{keV}}=10^{42.7}-10^{44.5} \mathrm{erg} \mathrm{s}^{-1}$ with a median of $10^{44.0} \mathrm{erg} \mathrm{s}^{-1}$. The AGNs include the entire range from entirely unobscured to highly obscured, and have moderate luminosities, and thus accretion rates, typical of the local universe. Quasars exhibit even higher luminosities and accretion rates, but are rare in the low-redshift universe; our sample probes almost all of the significant black hole growth in the local universe.

\section{HOST GALAXY COLORS AND CENTRAL POINT-SOURCE SUBTRACTION}

To study the host galaxy optical properties, we use imaging data from the SDSS DR6 (York et al. 2000; Adelman-McCarthy et al. 2008). The main challenge in understanding the host galaxy colors of AGNs is the contamination of the nonstellar central 

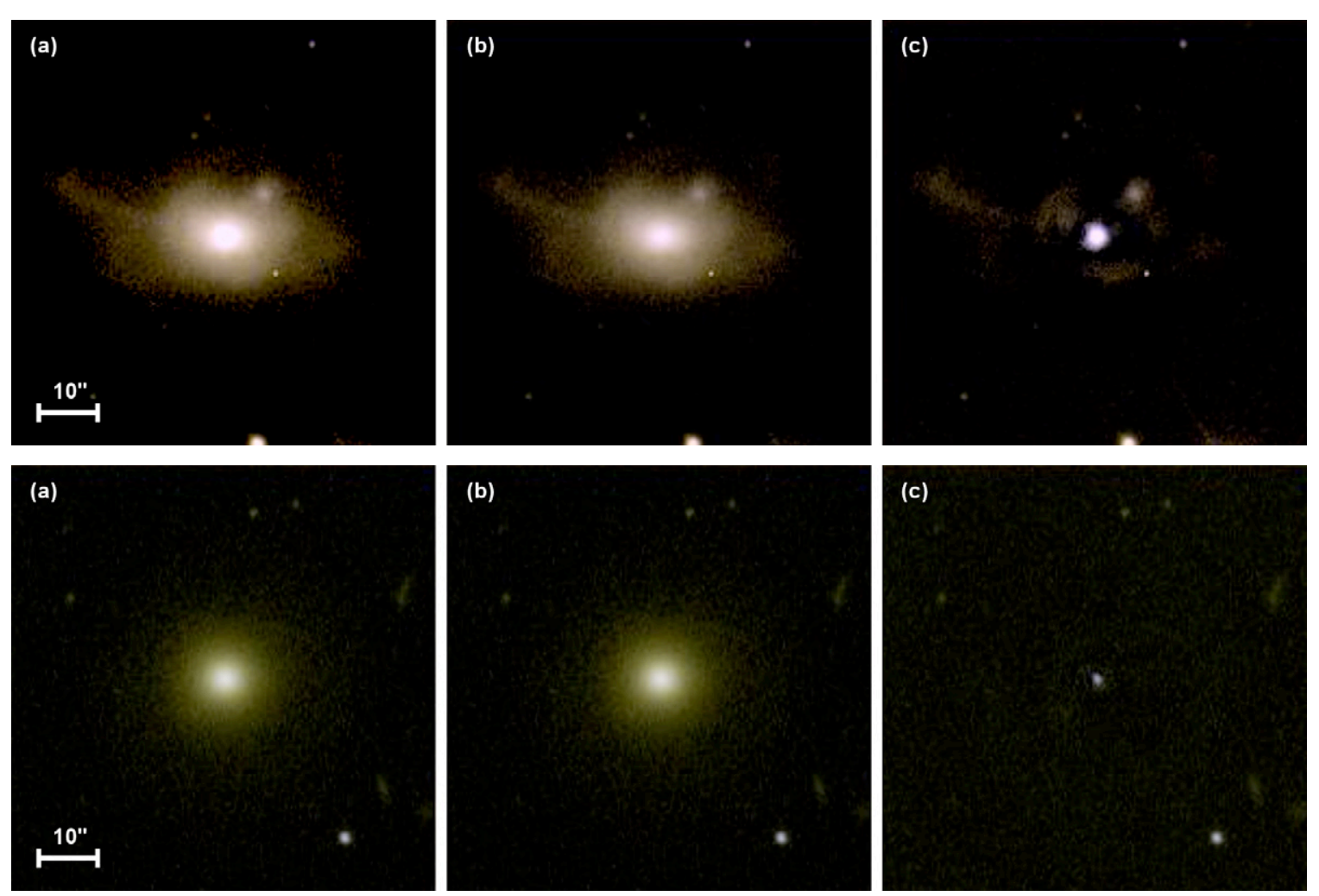

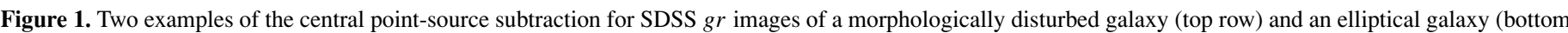

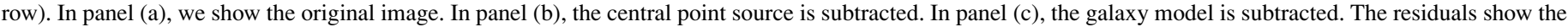
blue central point source, and in the case of the merger galaxy, tidal tails and shells.

point source of the AGNs. Deep X-ray surveys can deliver samples of X-ray-selected AGNs down to moderate X-ray luminosities out to considerable redshifts, but the contamination by the AGN light can make comparing AGN host galaxies to their normal counterparts meaningless (Simmons \& Urry 2008). At high redshifts $(z \gtrsim 0.7)$, it is difficult to separate host galaxy from central source even with the superb angular resolution of the Hubble Space Telescope (HST). At very low redshifts, however, ground-based optical imaging data become comparable to, even superior to, the best HST images of highredshift galaxies.

We use GALFIT version 2.0.3 (Peng et al. 2002), modified by Simmons \& Urry (2008) for the optimal removal of central point sources, to reliably and robustly separate the central AGN light from the extended host galaxy. In Figure 1, we show two examples of the results of this process. We remove from our sample any sources where the point source is brighter than the host galaxy and sources where the host half-light radius is less than 2 pixels in order to robustly measure the host galaxy colors down to $\sim 0.1 \mathrm{mag}$. This leaves us with 16 sources from the Swift BAT/SDSS sample. This criterion introduces a slight bias against the most luminous AGNs in the BAT sample $\left(L_{\mathrm{X}} \gtrsim 10^{44} \mathrm{erg} \mathrm{s}^{-1}\right)$.

\section{RESULTS}

\subsection{The Optical Colors of Normal Galaxies and Hard X-Ray-Selected AGN Hosts}

In order to compare AGN host galaxies to normal galaxies, we construct a volume-limited sample of galaxies from the SDSS in the same redshift range with $M_{r}<-19$ mag to select galaxies sampling the entire luminosity range of the AGN host galaxies.

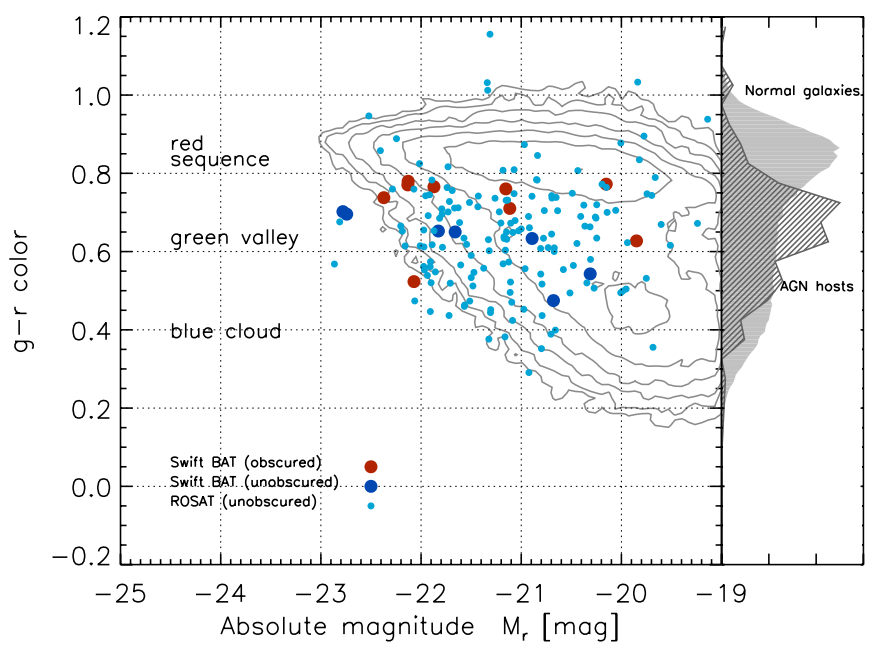

Figure 2. CMD for the host galaxies of X-ray-selected AGNs (filled points) and a matched sample of normal galaxies (contours, doubling at each level). The $g-r$ color is a good tracer of the age of the stellar population dominating the optical light. The galaxy comparison sample from SDSS clearly shows the red sequence and blue cloud. After removing the central point source, the X-rayselected AGNs appear in the green valley in between. The large points are hard $\mathrm{X}$-ray-selected AGNs detected by Swift BAT, colored according to obscuration $\left(\log N_{\mathrm{H}} \leqslant 22\right.$, blue; $\log N_{\mathrm{H}}>22$, red). The small points are the unobscured AGNs detected by ROSAT. On the right hand, we plot $g-r$ color histograms of both the galaxy comparison sample and the AGN sample.

In Figure 2, we show the CMD for the normal galaxy population, featuring the color bimodality of the red sequence, blue cloud, and the green valley in between. On top of this, we plot the host galaxy population of hard X-ray-selected AGNs (large filled circles). Obscured (red) and unobscured AGNs (blue) 
clearly reside in the green valley. No AGN of similar hard X-ray luminosity, regardless of obscuration, lies in the region typical of star-forming galaxies in the blue cloud.

We use a Kolmogorov-Smirnov (K-S) test to assess the significance of the difference between the host galaxy colors of the comparison sample and the AGN host galaxies and find that they are different at very high significance $(\gg 3 \sigma)$. In order to strengthen this statement, we compare the color distribution in luminosity bins. In all four bins of 1 mag each between $M_{r}=-23$ and $M_{r}=-19$, the $g-r$ color distribution of AGN host galaxies and of their normal counterparts are found to be significantly different at far more than the $3 \sigma$ level.

\subsection{Locating the Obscuring Material}

The unobscured AGNs show the same green host galaxy colors as the obscured AGNs, meaning the obscuration must be located in the nucleus, not distributed across the galaxy. A K-S test of the $g-r$ color distribution of the obscured and unobscured BAT sources yields that they are consistent with being drawn from the same parent distribution at the $2 \sigma$ level. The obscuration seen by the AGNs must therefore be circumnuclear, as in the Unified Model of AGNs, such that the galaxy colors are not strongly affected by the obscuration. Treister \& Urry (2006) showed that there is evidence that this may not be the case at higher redshifts, toward the peak of AGNs, and galaxy merger activity, implying evolution of the obscuration.

\subsection{The Optical Colors of Soft X-Ray-Selected AGNs}

Clearly, unobscured AGNs appear to have the same green host galaxy colors as obscured AGNs - but the sample is small. To improve statistics, we obtain a larger sample of unobscured AGNs using data from ROSAT (Voges et al. 1999; Anderson et al. 2007), which is sensitive in the $0.1-2.4 \mathrm{keV}$ energy range, and thus to mostly unobscured AGNs. Restricting ourselves to the same SDSS area and redshift range, we obtain a sample of 161 unobscured AGNs with soft X-ray luminosities of $L_{0.1-2.4 \mathrm{keV}}$ of $10^{42}-10^{44.3} \mathrm{erg} \mathrm{s}^{-1}$ and a median of $10^{42.9} \mathrm{erg} \mathrm{s}^{-1}$. We limit our selection to a minimum of $10^{42} \mathrm{erg} \mathrm{s}^{-1}$ to avoid contamination with X-rays from star formation and X-ray binaries. The energy ranges observed by Swift and ROSAT are different; however, assuming an unobscured AGN spectrum with $\Gamma=1.7$, the median luminosities of our ROSAT and Swift BAT samples are comparable within a factor of 3. A K-S test affirms that they are consistent with being drawn from the same parent distribution, but note that the Swift BAT sample is small.

We again subtract the optical central point sources and add the remaining 161 sources with robust colors to Figure 2 (small blue points). Like the unobscured AGNs detected by Swift BAT, the ROSAT-detected AGNs have green host galaxy optical colors. The larger numbers allow us to probe the bluest and reddest colors of AGN host galaxies. and in particular, to see how many objects lie in the blue cloud. We see no AGN in the peak of the blue star-forming galaxy population (lower right of Figure 2). Due to the range of obscuration represented in our sample, this statement must hold for all local moderate-luminosity AGNs.

Could we be systematically missing accreting black holes at the lowest galaxy mass covered by our sample at $M_{r} \sim-19$ ? At the bluest colors, this corresponds to $\sim 10^{10} M_{\odot}$. We convert this to a black hole mass (Häring \& Rix 2004) and assume that it radiates at efficiencies as low as a few percent of the Eddington

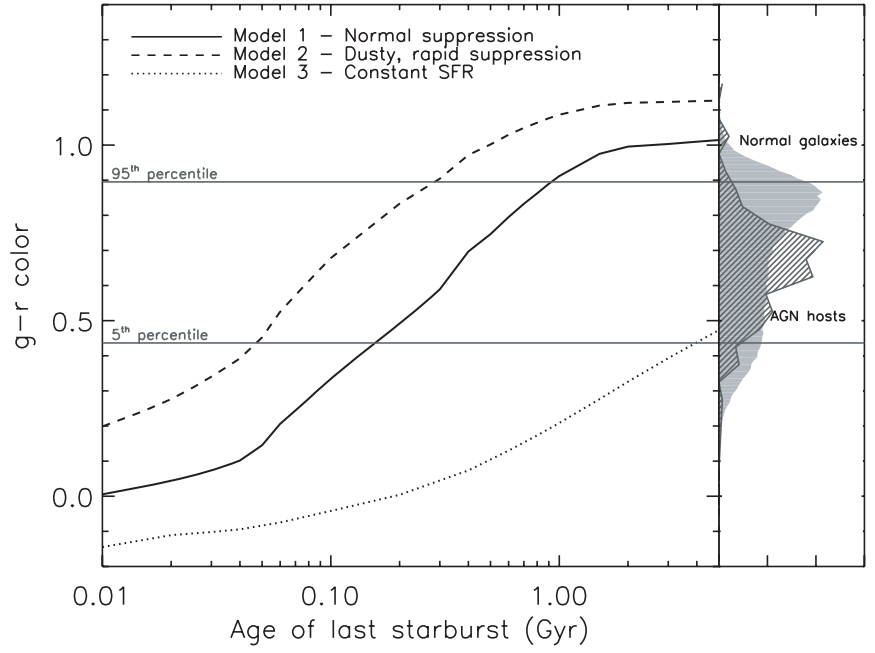

Figure 3. Timescales for "green" optical colors of AGN host galaxies. We plot three star formation scenarios probing the limits of possible color evolution based on stellar evolution models (Maraston 2005). Model 1 is a moderate $10 \%$ mass-fraction starburst on top of an old $8 \mathrm{Gyr}$ stellar population. This burst is somewhat extinguished with $E(B-V)=0.1$ and declines with an e-folding timescale of $100 \mathrm{Myr}$. Model 2 represents an extreme scenario of a small 5\% mass fraction declining effectively instantaneously, with $\tau=$ $10 \mathrm{Myr}$, extinguished with the maximum allowable $E(B-V)=0.2$. Model 3 has a constant star formation history spanning $10 \mathrm{Gyr}$ with $E(B-V)$ of 0.05 ; such galaxies never leave the blue cloud in most cases. That $95 \%$ of AGN host galaxies lie above $g-r=0.45$ means that Model 3 is ruled out definitely. Models 1 and 2 are consistent with our results, but imply implausibly long AGN lifetimes unless there is a significant time delay between the suppression of star formation and the onset of detectable AGN activity.

limit. Such an AGN would emit a soft X-ray luminosity of $\sim 10^{42} \mathrm{erg} \mathrm{s}^{-1}$ and thus should have been detected by ROSAT. The lack of blue AGN hosts is therefore significant and we conclude that the majority of AGN host galaxies in the local universe are in the green valley.

\subsection{A High Merger Fraction for X-Ray-Selected AGN Host Galaxies}

After subtracting the galaxy model with GALFIT, the residual image contains both the central AGN point source, and any remaining morphological disturbances, such as tidal tails, spiral arms, and shells (e.g., Figure 1, top). We perform the same galaxy model subtraction to a sample of normal galaxies matched to the AGN sample in both $g-r$ color and luminosity, and compare the incidence of features indicating a recent or in-progress merger in both. We find a statistically highly significant excess of such features in the AGN host galaxy sample over the control sample $(43 \%$ for BAT sources and $21 \%$ for control). We further measure the asymmetry (Conselice 2003 ) in both the $g$ - and $r$-band images and find that the AGN host galaxies are significantly more asymmetric than those in the control sample. This observation compares favorably to models in which AGN phases are driven by mergers (e.g., Hopkins et al. 2006).

\section{STELLAR POPULATION ANALYSIS}

We can use stellar population synthesis as a clock to interpret the green valley optical colors. In Figure 3, we plot $g-r$ colors of three representative star formation histories as a function of time, based on the stellar population models of Maraston (2005). Models 1 and 2 trace the evolution of realistic and extreme star formation shutdown scenarios, respectively, and Model 3 
follows a constant star formation rate (SFR). Varying the model parameters such as mass fraction, dust, and timescale $\tau$ fills in the parameter space between Models 2 and 3, clustering around the "normal" Model 1.

A particular concern is whether we can account for the green color of AGN host galaxies by invoking substantial ongoing, but obscured, star formation. Any extinction seen by the host galaxy would also be seen by the central engine. The unobscured AGNs detected with ROSAT all have column densities of $N_{\mathrm{H}}<10^{22} \mathrm{~cm}^{-2}$. Converting this column density to a dust extinction using the observed ratio of Bohlin et al. (1978), $A_{\mathrm{V}}=0.58\left(\frac{N_{\mathrm{H}}}{10^{21}}\right)$, yields a maximum value for the optical extinction $E(B-V)$ of $\sim 0.2$ for a typical $R_{\mathrm{V}}=3.1$. Substantial ongoing star formation requires sufficient amounts of dust, but such high levels of dust obscuration cannot be present in our AGN host galaxies.

With Model 2, we put a lower bound on the transition time from the blue cloud to the green valley. Longer suppression timescales directly result in longer time delays to the green valley. In Model 1 , the $\tau$ of $100 \mathrm{Myr}$ is comparable to the dynamical timescale of massive galaxies and thus sets a natural timescale that compares favorably with observed shutdown timescales inferred in post-starburst galaxies (e.g., Kaviraj et al. 2007a; Wild et al. 2008) and for molecular gas reservoir destruction (Schawinski et al. 2009). The extreme Model 2 assumes virtually instantaneous suppression ( $\tau=10 \mathrm{Myr})$.

The mass fraction of young stars is also a factor in estimating the transition time. The mass fraction of young stars for the extreme Model 2 of $5 \%$ is chosen to reflect a minor starburst; smaller starbursts at the sub-1\% level are widespread, but fail to move red galaxies off the optical red sequence (Yi et al. 2005; Schawinski et al. 2007b; Kaviraj et al. 2007b). While there are still parameter choices that might give green optical colors at very young ages, such fine tuning seems implausible for our sample of almost 200 objects. The mass fractions chosen for the extreme $(5 \%)$ and realistic $(10 \%)$ models are commensurate to those in green valley early-type galaxies transitioning from the blue cloud to the red sequence (Schawinski et al. 2007a).

For the extreme Model 2, the $g-r$ colors of the AGN host galaxies restrict the minimum time elapsed since the suppression event to $\sim 60 \mathrm{Myr}$, as only the bluest $5 \%$ of the population exhibit colors that correspond to younger ages. For the more realistic Model 1, the bluest 5\% imply typical ages of a few hundred Myr.

\section{DISCUSSION}

The fact that we detect very few AGNs in the peak of the blue cloud seems to rule out scenarios where a short-lived, luminous AGN/quasar phase episode suppresses star formation on short timescales (i.e., Scenario A (red) in Figure 4).

Two scenarios instead can account for the lack of young, blue AGN host galaxies. The first is that the AGNs suppress star formation in their hosts, but the typical AGN lifetime is long compared to the suppression timescale, so that most AGNs have evolved away from the blue cloud (Scenario B (green) in Figure 4). Roughly 5\% of our AGNs still have ages less than $\sim 60 \mathrm{Myr}$, in which case Scenario B implies a lifetime of $\gtrsim 1.2$ Gyr. However, the estimates of Marconi et al. (2004) reach $\gtrsim 1$ Gyr only for the most massive black holes. The current consensus in the literature (e.g., Martini \& Weinberg 2001; Martini 2004; Marconi et al. 2004) gives typical lifetimes of only a few $10^{8}$ years, which are smaller than the minimum lifetime implied by the dearth of blue host galaxies. Such

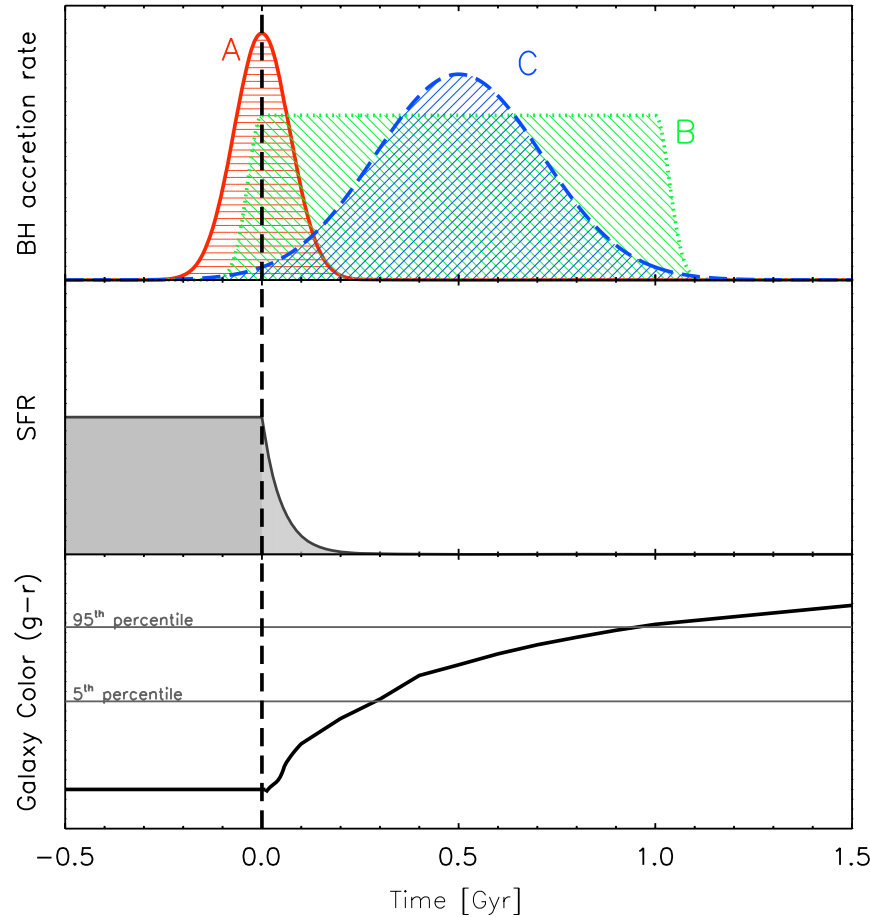

Figure 4. Black hole accretion rate, SFR, and optical color as a function of time, for the case of rapid ( $\tau=100 \mathrm{Myr}$ ) suppression of star formation. In the top panel, we show three scenarios for the black hole accretion rate. Scenario A (red, solid) represents a short-lived AGN/quasar phase that suppresses star formation instantly. This kind of scenario would predict a large number of AGNs in the blue cloud, and so is ruled out by our observations. In Scenario B (green, dotted), the AGN quickly switches on, suppresses star formation, and remains at high luminosity for $\sim 1 \mathrm{Gyr}$ to account for the high fraction of AGNs in the green valley compared to the blue cloud. In Scenario C (blue, dashed), the moderate luminosity AGNs (like those we detect in the X-rays) does suppress star formation, but peaks in luminosity several 100 Myr after the suppression, naturally giving rise to the observed distribution of AGN host galaxy colors. Such large time delays have been seen in some cosmological simulations (e.g., Li et al. 2007), although these are for high-luminosity quasars at very high redshift.

lifetime estimates are generally given for accretion phases with high Eddington ratios, with the estimate of a few $10^{8}$ years of Marconi et al. (2004) considering efficiencies down to $0.1 L_{\text {Edd }}$. The median Swift BAT source in our sample has efficiencies of $L \sim 0.1 L_{\text {Edd }}$ with a large scatter if we compute $L / L_{\text {Edd }}$, for black hole masses estimated from the $M_{\bullet}-\sigma$ relation (Ferrarese $\&$ Merritt 2000; Gebhardt et al. 2000) and apply a bolometric correction from $L_{\mathrm{X}}$ to $L_{\mathrm{bol}}$ of $20-100$.

The other interpretation (Scenario C (blue) in Figure 4) is that star formation is suppressed immediately, but the AGN luminosity is detectable only with a delay of about $100 \mathrm{Myr}$. If so, low-luminosity AGNs during the rising phase, below $\sim 10^{42} \mathrm{erg} \mathrm{s}^{-1}$, must be sufficient to shut down star formation, as suggested by the destruction of cold molecular gas reservoirs by low-luminosity AGNs (Schawinski et al. 2007a, 2009). Then the optical colors of the green valley AGNs are a reflection of the time needed for substantial amounts of material to lose enough angular momentum to reach the black hole and thus increase the AGN luminosity. This time gap of $\sim 100$ Myr cannot be accounted for by a heavily obscured AGN phase, as Swift BAT would have detected them. In this Scenario C, the lowluminosity phase and not the X-ray-bright phase is the cause of the shutdown of star formation. Perhaps the low-luminosity phase, while radiatively inefficient, is channeling substantial amounts of kinetic energy into the interstellar medium (ISM), similar to the "radio" or "maintenance" mode invoked in current 
semianalytical models (Bower et al. 2006; Croton et al. 2006; Somerville et al. 2008). This scenario also leaves the option that another process causes both the shutdown of star formation and the AGN phase with a time delay, but such a scenario raises more problems than it solves.

Our observational result challenges recent results from simulations of AGN feedback, at least at low redshifts (Springel et al. 2005; Di Matteo et al. 2005; Hopkins et al. 2006; Johansson et al. 2009), which suggest that the epoch of maximum black hole growth is coeval with the peak of star formation. The coeval scenario (i.e., Scenario A), where the AGN luminosity peaks during the suppression phase, would predict a large population of high-luminosity AGNs in the blue cloud, where we observe very few. We note however that some of these simulations pertain to the high-redshift universe, and that the physical processes at low redshift may be different.

We thank Joseph Silk, Yuexing Li, and Sukyoung Yi for comments and discussions. S.V. acknowledges support from a graduate research scholarship awarded by the Natural Science and Engineering Research Council of Canada (NSERC) and from NASA/INTEGRAL grant NNG05GM79G. B.S. and C.M.U. received support from NSF grant AST0407295. Support for E.T. was provided by the National Aeronautics and Space Administration through Chandra Postdoctoral Fellowship Award Number PF8-90055 issued by the Chandra X-ray Observatory Center, which is operated by the Smithsonian Astrophysical Observatory for and on behalf of the National Aeronautics Space Administration under contract NAS8-03060. S.K. acknowledges a research fellowship from the Royal Commission for the Exhibition of 1851. This publication is based on data from the Sloan Digital Sky Survey, the Swift Gamma Ray Burst Telescope and the Röntgen Satellite (ROSAT).

Facilities: Swift (BAT), ROSAT, Sloan

\section{REFERENCES}

Adelman-McCarthy, J. K., et al. 2008, ApJS, 175, 297

Anderson, S. F., et al. 2007, AJ, 133, 313
Antonucci, R. 1993, ARA\&A, 31, 473

Bohlin, R. C., Savage, B. D., \& Drake, J. F. 1978, ApJ, 224, 132

Bower, R. G., et al. 2006, MNRAS, 370, 645

Bundy, K., et al. 2008, ApJ, 681, 931

Conselice, C. J. 2003, ApJS, 147, 1

Constantin, A., Hoyle, F., \& Vogeley, M. S. 2008, ApJ, 673, 715

Croton, D. J., et al. 2006, MNRAS, 365, 11

Di Matteo, T., Springel, V., \& Hernquist, L. 2005, Nature, 433, 604

Ferrarese, L., \& Merritt, D. 2000, ApJ, 539, L9

Gebhardt, K., et al. 2000, ApJ, 539, L13

Gehrels, N., et al. 2004, ApJ, 611, 1005

Georgakakis, A., et al. 2008, MNRAS, 385, 2049

Häring, N., \& Rix, H.-W. 2004, ApJ, 604, L89

Hopkins, P. F., Hernquist, L., Cox, T. J., Di Matteo, T., Robertson, B., \& Springel, V. 2006, ApJS, 163, 1

Johansson, P. H., Naab, T., \& Burkert, A. 2009, ApJ, 690, 802

Kaviraj, S., Kirkby, L. A., Silk, J., \& Sarzi, M. 2007a, MNRAS, 382, 960

Kaviraj, S., et al. 2007b, ApJS, 173, 619

Li, Y., et al. 2007, ApJ, 665, 187

Maraston, C. 2005, MNRAS, 362, 799

Marconi, A., Risaliti, G., Gilli, R., Hunt, L. K., Maiolino, R., \& Salvati, M. 2004, MNRAS, 351, 169

Martini, P. 2004, in Coevolution of Black Holes and Galaxies, ed. Ho L. C. (Cambridge: Cambridge Univ. Press), 169

Martini, P., \& Weinberg, D. H. 2001, ApJ, 547, 12

Nandra, K., et al. 2007, ApJ, 660, L11

Peng, C. Y., Ho, L. C., Impey, C. D., \& Rix, H.-W. 2002, AJ, 124, 266

Salim, S., et al. 2007, ApJS, 173, 267

Schawinski, K., Thomas, D., Sarzi, M., Maraston, C., Kaviraj, S., Joo, S.-J., Yi, S. K., \& Silk, J. 2007a, MNRAS, 382, 1415

Schawinski, K., et al. 2007b, ApJS, 173, 512

Schawinski, K., et al. 2009, ApJ, 690, 1672

Silverman, J. D., et al. 2008, ApJ, 675, 1025

Simmons, B. D., \& Urry, C. M. 2008, ApJ, 683, 644

Somerville, R. S., Hopkins, P. F., Cox, T. J., Robertson, B. E., \& Hernquist, L. 2008, MNRAS, 391, 481

Springel, V., Di Matteo, T., \& Hernquist, L. 2005, MNRAS, 361, 776

Treister, E., \& Urry, C. M. 2006, ApJ, 652, L79

Tueller, J., Mushotzky, R. F., Barthelmy, S., Cannizzo, J. K., Gehrels, N., Markwardt, C. B., Skinner, G. K., \& Winter, L. M. 2008, ApJ, 681, 113

Urry, C. M., \& Padovani, P. 1995, PASP, 107, 803

Voges, W., et al. 1999, A\&A, 349, 389

Wild, V., Walcher, C. J., Johansson, P. H., Tresse, L., Charlot, S., Pollo, A., Le Fevre, O., \& de Ravel, L. 2008, arXiv:0810.5122

Yi, S. K., et al. 2005, ApJL, 619, L111

York, D. G., et al. 2000, AJ, 120, 1579 